J. Comp. Int. Sci. (2016) 7(2):03-24

http://epacis.netjicis/PDF_JCIS/JCIS-0106.pdf

jcis@epacis.net

@2016 PACIS (http://epacis.net)

\title{
Comparison of Space Weather Services: Information Systems, Activity and Forecasts
}

\author{
Fernando J. G. Pinheiro ${ }^{a 1}$, M. T. Barata ${ }^{a}$ and J. M. Fernandes ${ }^{a, b}$ \\ ${ }^{a}$ CITEUC, Geophysical and Astronomical Observatory, University of Coimbra, 3040-004, Coimbra, Portugal \\ ${ }^{b}$ Department of Mathematics, University of Coimbra, 3001-454, Coimbra Portugal
}

Received on August 20, 2016 / accepted on August 31, 2016

\begin{abstract}
Space Weather (SW) is a field of Space Sciences which deals with the variable conditions in the Sun, the interplanetary medium and our planet's vicinity, which have an impact on human safety and technological systems. In this work we compare the information provided by several SW Information Systems, with a particular emphasis on the activity indices used to describe the SW conditions. For that purpose we analyse the daily SW conditions and forecasts for the year 2015. From this study we conclude that the SW services evaluated here present similar levels of forecast accuracy, predicting the correct SW conditions in 70 to $90 \%$ of the cases. Despite that, even when the forecasts of two warning centers are correct, differences in the activity scales used by each institution can result in divergent reports.
\end{abstract}

Keywords: space weather, space weather indices, forecasting, validation, information systems.

\section{Introduction}

In modern societies space weather (SW) phenomena can have a huge social and economic impact [1, and references therein]. High frequency communications can be disrupted by solar flares and geomagnetic disturbances. The solar wind can affect global navigation systems, which are nowadays important for the transportation of people and goods. Likewise, satellites can be incapacitated by high energy solar particles and geomagnetic storms [2,3]. On March $13^{\text {th }} 1989$ the Canadian region of Quebec suffered a sudden blackout caused by intense solar activity which took place three days earlier. This blackout lasted for nine hours, affecting millions of people and had a total cost of 13.2 million dollars [4]. Even smaller disturbances caused by SW events can result in the loss of human lives. During the battle of Takur Ghar in Afghanistan ( $4^{\text {th }}$ of March of 2002) a Chinook rescue helicopter crash-landed on a territory under enemy control as a consequence of satellite communication difficulties. Subsequent analysis found evidences that UHF outages may have been caused by intense solar activity [5].

Space weather events are affecting activities that we consider ordinary. Between 15 and 19 of January of 2005 flights between Chicago and Hong Kong had to be deviated from the usual routes across the polar regions since this part of the globe is particularly vulnerable to solar activity, affecting navigation and communication systems. This lead to an increase of the duration of flights (around three extra hours) and the need of an extra refuelling stop, resulting in additional costs to airlines operating those routes [6]. Likewise on December of 2006 a strong solar flare caused a considerable perturbation on the Global Positioning System's (GPS) signal, affecting the operations of deep-ocean drilling platforms. The daily operation costs of these infrastructures can reach a million of dollars [6]. Intense solar activity was also responsible for the

\footnotetext{
${ }^{1}$ E-mail Corresponding Author: fjgpinheiro.astro@gmail.com
} 
degradation of the European Geostationary Navigation Overlay Service's (EGNOS) signal between the $20^{\text {th }}$ and $28^{\text {th }}$ of October of 2009 [7].

Extreme SW events can have far greater consequences. The impact of a Carrington level geomagnetic storm [8] to the North American electric grid could cost between 0.6 and 2.6 trillion US Dollars [9]. This amount is more than the annual national gross product of some small countries. In addition, the replacement of the damaged high voltage power transformers could take several months. This situation will get even worse as we grow more dependent of these technological systems. Indeed as stressed by Schrjver [1]: "there is an urgent need to improve forecasts in order to reduce demonstrated impacts caused by severe space weather and the considerable concerns regarding more severe events". Several countries created their own infrastructures for the monitoring and forecast of SW events having this problem in mind. Among the top SW services are the National Oceanic and Atmospheric Administration's (NOAA) Space Weather Prediction Center (SWPC), the Australian Space Weather Services (SWS), the Belgian Solar Influences Data Analysis Center (SIDC), the Korean Space Weather Center (KSWC), and the Japanese National Institute of Information and Communications Technology (NICT). International organisations such as the European Space Agency's (ESA) and the World Meteorological Organisation (WMO) are also involved in this issue. Several of these institutions cooperate in international projects and consortia such as COSPAR (Committee for Space Research), ISES (International Space environment Services), COMESEP (Coronal Mass Ejections and Solar Energetic Particles), EURISGIC (European Risk from Geomagnetically Induced Currents) or the ILWS (International Living with a Star). The commercial sector is also responding to this crescent need for particular SW applications, including companies such as the Seibersdorf Laboratories, Atmospheric \& Space Technology Research Associates (ASTRA), the Space Environment Corporation (SEC) and associations such as the American Commercial Space Weather Association [10].

Several organisations use their own monitoring and forecast strategies, presenting different quantities associated with the diverse types of SW activity. Moreover, many institutions use systems of scales to facilitate the communication of present and future SW conditions to the general public. These scales are based on the impacts of each activity level. However not all SW services use the same scales.

Despite monitoring/forecast tools and the aforementioned activity scales have been extensively validated e.g. [11, 12, 13, for instance], it is important to compare the information provided by different SW services. Particularly we need to know if the information supplied by these institutions is consistent with each other. The most notable work done in this respect was carried out by NICT (www2.nict.go.jp/ aeri/swe/swx/swcenter/isesforecast_e.html and http://seg-web.nict.go.jp/cgi-bin/forecast/eng_forecast_score.cgi). Yet this analysis is only focused in accuracy of the forecasts, disregarding the different scales used by each institution and without comparing the forecasts of solar energetic particle storms. Moreover, NICT only presents the forecasts of SW conditions. Therefore, from their tool one does not get a general comparison of the type of information that is made available by SW warning centers. To the best of our knowledge there is no such data comparison is available in the literature.

In this work we will start by analysing information provided by several SW services, ranging from some of the largest institutions in this field to regional warning centers. Section 3 provides a comparison of the indices used in the characterisation of the SW activity degree. In the following section (4) can be found a brief description of tools and methods used to predict the SW conditions. Finally, Sections 5 and 6 present an analysis of the forecast accuracy of several SW services, i.e. their ability to predict the correct activity level for the next day. The later evaluation corresponds to an expansion of NICT's forecast comparison by adding the analysis solar proton forecasts and by adding the forecasts of the Brazilian EMBRACE (Estudo e Monitoramento Brasileiro do Clima Espacial) and the Czech IAP (Institute of Atmospheric Physics) for the year 2015 .

\section{Information Systems provided by Space Weather Services}

From the comparison of several SW warning centers across the globe, including those presented at Table 1, one can notice that SW services present a set of complementary observables and models valuable for understanding past, present and future SW conditions. The diversity and amount of data involved fall within the Big Data definition. 


\subsection{Big Data and Computational Resources}

The information required and provided by SW services can be subdivided in three main categories: solar data, properties of the interplanetary medium and near earth vicinity and geophysical data (including magnetospheric and ionospheric parameters). In the following paragraphs we mention some of the observational and post-processed data provided by SW services.

Satellite (SOHO, Hinode, SDO, GOES 13 \& 15, Proba2, HESSI) and ground based observatories (e.g.: UCLE, Culgoora, GONG, etc.) provide solar observations in multiple wavelengths, ranging from radio to X-rays. Computational tools such as ASSA and ASAP are used to identify/characterise solar active regions including sunspots, filaments, coronal holes and plages, thus deriving the sunspot number, an important indicator of solar activity. Other tools such as CACTUS, SoFast and SolarDemon allow identifying the occurrence of flares and coronal mass ejections and estimate their possible impact. Radio observatories such as the Culgoora (Australia), Nançay (France) observatories or the Icheon (Korea) and Humain (Belgium) radio astronomy stations are used to monitor the solar radio emission in multiple wavelengths. In this respect, a particular highlight should be given to the Dominion Radio Astrophysical Observatory (Canada), a reference institution for the determination of the $10.7 \mathrm{~cm}$ solar radio flux (F107), another important indicator of solar activity.

At the same time, satellites such as ACE and STEREO are used to characterise the solar wind in terms of speed, density, temperature, magnetic field strength and orientation. Radio observatories are also used to measure the interplanetary scintillation (e.g. Icheon station), another method of characterising the solar wind. The WSA-Enlil model [14, 15] combines solar wind data with the characterisation of CMEs, to produce a predictive physical model of the heliosphere which allows to identify with four days in advance the CME's and solar wind structures capable of causing geomagnetic storms. Likewise the Drag-Based Model (DBM) of Vrs̆nak et al. (2013) uses CME information and solar wind properties to estimate the arrival times of these CMEs. On the other hand, solar wind information from the ACE satellite is used by Relativistic Electron Forecast Model (REFM) to predict electron fluencies at geo-synchronous orbit, while KSWC's Solar Proton Event Flux Prediction Model analyses X-ray fluxes from solar flares, characterises CMEs predicts the intensity of proton fluxes. This information can be used to compute auroral maps such as the ones provided by the Ovation code [16]. Finally the GOES 13 and 15 satellites provide information about proton and electron fluxes at geo-stationary orbits.

Geomagnetic data is obtained from stations around the world. Many of these stations are part of vast networks of observatories such as INTERMAGNET (http://www.intermagnet.org/). Magnetograms from these stations include the three components of the magnetic field and their derivatives. A particular attention is given to the horizontal component of the magnetic field $(\mathrm{H})$ since this value and its temporal derivative $(\mathrm{dH} / \mathrm{dT})$ are used to evaluate the occurrence of geomagnetic storms and geomagnetically induced currents (GICs). Generally GIC maps are computed on a national or regional scale. Variations of the geomagnetic horizontal component measured from observatories located at low latitudes are used to compute the Disturbance Storm Time index (Dst). Large geomagnetic storms cause a high altitude ring equatorial currents which generate a decrease in this index. Some institutions compute their own disturbance storm time indices based on local data (e.g. SWS, \& SeNMEs). Nevertheless the most used Dst index is provided by Kyoto's World Data Center (WDC) for geomagnetism. Additionally the horizontal component of the geomagnetic field is used to compute local $\mathrm{K}$ and $\mathrm{A}$ indices, which are also indicators of the occurrence of geomagnetic storms. Some institutions combine data from several observatories located at intermediate geomagnetic latitudes to obtain planetary $\mathrm{K}$ and A indices (Kp \& Ap respectively). NOAA's SWPC and the German Research Centre for Geosciences (GFZ), provide some of the Kp indices most used worldwide. Other geomagnetic indices, such as the auroral electro-jet (AE) index, can also be provided. Moreover, ionospheric parameters are derived from ionosondes across the world. Among other properties some SW services provide local measurements of the Maximum Usable Frequency (MUF), the critical frequencies of the $\mathrm{F} 2$ and $\mathrm{E}$ ionospheric layers (FoF2 \& FoE), the T-index (indicator of the highest frequencies that are able to be refracted at the ionosphere), Total Electron Content (TEC) and ionospheric scintillation index (S4). Global positioning stations can also be used to derive the TEC and S4 parameters [17, for instance]. These observations allow computing regional and global TEC, T-index, scintillation and Short Wave Fadeout 
(SWF) maps.

A general trend among several SW services is the use of data from local/national sources. This makes particular sense given the regional impact of many SW events. The frequency rate at which different observables are acquired and models produced varies greatly. While some ground-based observatories such as the Coimbra Geophysical and Astronomical Observatory (OGAUC) acquire solar observations on a daily basis (a sampling interval identical to the one in which the sunspot number is computed), the SOHO satellite acquires an image of the Sun every 12 minutes. Moreover, while GOES 15's X-ray imager takes an image of the Sun every minute, the SDO satellite does the same in a second. On the other hand, radio observations of the Sun (for instance with the CALLISTO spectrometer [18] have temporal resolutions of the order of milliseconds. Yet in many cases we only get a daily F107 solar radio flux. Some of the data obtained from instruments in orbit (e.g. solar wind parameters, x-ray and energetic particle fluxes) tend to be presented in 1 minute, 5 minutes, 1 hour or daily averaged intervals. This is well bellow the frequency rate at which the observables are acquired (above the $\mathrm{Hz}$ ). Geomagnetic observations are usually obtained within a 1 second to 1 minute interval while, by definition, DST ad planetary $\mathrm{K}$ indices are computed within 1 and 3 hour intervals respectively. At the same time ionograms and TEC maps are produced every 10 to 15 minutes, while GIC maps and the Ovation Aurora Short Term Forecasts are updated every 5 minutes.

Many of these observations are presented in near real-time (i.e. just a few minutes after they are acquired), yet one should take into account that in the case of geomagnetic observations, the final calibration of data is only achieved in the following year.

In addition to different sampling intervals these observations, models, and maps have a wide range of sizes. For instance, while OGAUC's daily 1 Mpixel observations in JPG format occupy about $80 \mathrm{kB}$ of disk space, SDO's 16 Mpixel files occupy 2.3Mb. The volume of information increases furthermore when dealing with uncompressed data formats: the size of OGAUC's observations in the FITS format goes up to $1.9 \mathrm{Mb}$. Moreover, the raw daily spectrographic observations of the Culgoora and Learmonth observatories (Australia) occupy about $27 \mathrm{MB}$ of disk space each. On the other hand, the daily 5 -minute averaged solar particle and electron fluxes from each one of the GOES satellites occupy about $36 \mathrm{kB}$ of disk space. It is not difficult to imagine the full size occupied by the annual 1-minute averaged data from both GOES 13 and 15 satellites (with without taking into account their raw data). Nevertheless, if we only consider the daily characterization of solar energetic particle events, we can decrease the size of each file to a couple $\mathrm{kB}$ each. This is what happens with data from the Hinode Flare Catalogue [19]. A similar reasoning can be made in respect to satellite data such as ACE's solar wind parameters. In what concerns the geomagnetic data, each one the $77 \mathrm{kB}$ ASCII files of the daily 1-minute sampled observations of OGAUC are larger than the 24kB annual file containing Kp indices of the Helmholtz-Zentrum Potsdam (GFZ-Potsdam) World Data Center, which comes as no surprise since the later ones are computed over 3 hour intervals. That value may not seem much, yet if we think about sampling intervals of the order of a second we reach to an annual output of 1.6Gb. Not to mention that several SW services take into account the geomagnetic observations of multiple stations. The same conclusion can also be drawn for cosmic ray data. Additionally, dealing with GPS data (for instance GNSS or ionospheric scintillation data) involves daily files of the order of some hundreds of $\mathrm{kB}$.

To all this data we should add the dozens of MB of TEC, a GIC and aurora maps and movies provided by some SW information systems such as the ones of NOAA/SWPC and SWS, plus the couple $\mathrm{kB}$ of the alarms and daily bulletins generated some SW services.

While some observables are easier to store due to their small volume, other information requires fully dedicated computational facilities. For instance, the Terabytes of data from space missions such as SoHo, ACE or SDO are stored in NASA's Space Physics Data Facility (http://spdf.gsfc.nasa.gov/ ). Moreover, several institutions possess the infrastructures required to store and distribute their observations, and those of their collaborators (solar, ionospheric, magnetic, etc.), as World Data Centers. Several observatories share their data in common repositories. As an example, OGAUC's solar observations is available at the BASS2000 database (bass2000.obspm.fr/) and the Global High-Resolution $\mathrm{H} \alpha$ Network (www.bbso.njit.edu/Research/ 
Halpha/). Likewise, data from geomagnetic stations across the globe can be found in Edinburgh's World Data Centre for Geomagnetism (www.wdc.bgs. ac.uk/index.html), including those of OGAUC (under the International Association of Geomagnetism and Aeronomy's designation: COI), or at the Intermagnet network (www.intermagnet.org/). It is worth to notice that some institutions have data archives in which observations, models, bulletins forecasts and alerts are made available. Several tools have also been developed for the visualization of large amounts of data currently available. JHelioviewer [20] is a visualisation software for accessing and viewing large archives of solar images. Likewise SWS provides tools for the visualization of the $\mathrm{H} \alpha$ images, radio spectrograms and ionograms provided by them.

Table 1: Information provided by space weather services across the world. National Oceanic and Atmospheric Administration (NOAA)/ Space Weather Prediction Cente (SWPC)

URL: http://www.swpc.noaa.gov Country: USA

Solar data: images (SOHO, GOES), sunspot number, F107, X-ray fluxes

(GOES), identification of active regions

Interstellar medium: energetic particle fluxes at geostationary orbit (GOES); solar wind speed, density, temperature, magnetic field strength (ACE)

Geomagnetic data: Kp index

Ionospheric data: TEC maps for continental USA

Forecasts: flare, geomagnetic \& solar radiation storm within 24,48 \& 72 hours

Dashboards for specific industries (aviation, electric power, satelite navigation radio communications, etc.)

Archive of warnings, alerts daily bulletins, weekly reports and data

Tools/models: WSA-Enlil - solar wind modelling [14, 15]. Wing-Kp - Kp index forecast [21]. Relativistic Electron Forecast Model (REFM) [22]. Ovation - aurora forecast [16]. D Region Absorption Predictions (D-RAP) [22].

Australian Bureau of Meteorology / Space Weather Services* (SWS)

http://www.ips.gov.au Country: Australia

Solar data: H-alpha, white light, magnetograms and radio spectrographs

(Culgoora and Learmonth solar observatories) X-ray (GOES) and solar

flare monitoring, Sunspot number and $10.7 \mathrm{~cm}$ solar radio flux

Solar energetic particle fluxes (GOES), solar wind speed \& magnetic field strength (ACE)

Planetary and Fredericksburg (USA) K indices, magenetograms, A and local DST indices from stations across Australia and Antarctica

Maximum usable frequency,

Cosmic ray fluxes at Kingston (Tasmania) and Mawson (Antarctica)

Alerts: energetic particle fluxes at geostationary orbits, geomagnetic, high frequency $(\mathrm{HF})$ communications and aurora occurrence

Selected data specific for particular clients (emergency services, aviation companies, radio communications)

Forecast of flare, geomagnetic, ionospheric and radio HF propagation conditions for the next 3 days

Archive of daily reports

Tools/models: Solar wind [23] and magnetopause [24] models. Auroral oval toool GEOSAT Discharge Model for electron fluency forecasts [25]. Ionospheric, FoF2, TEC and radio fade-outs world and regional maps. $\mathrm{K}$ and pc3-indices (magnetic field variations), scintillation T-index, GPS positioning errors and GIC maps for Australia. Radio propagation forecast tools: Ground Wave \& Advanced Stand Alone Prediction System (ASAPS) and Ground Wave Prediction Systems (GWPS) [26] 
Table 1: (continuation)

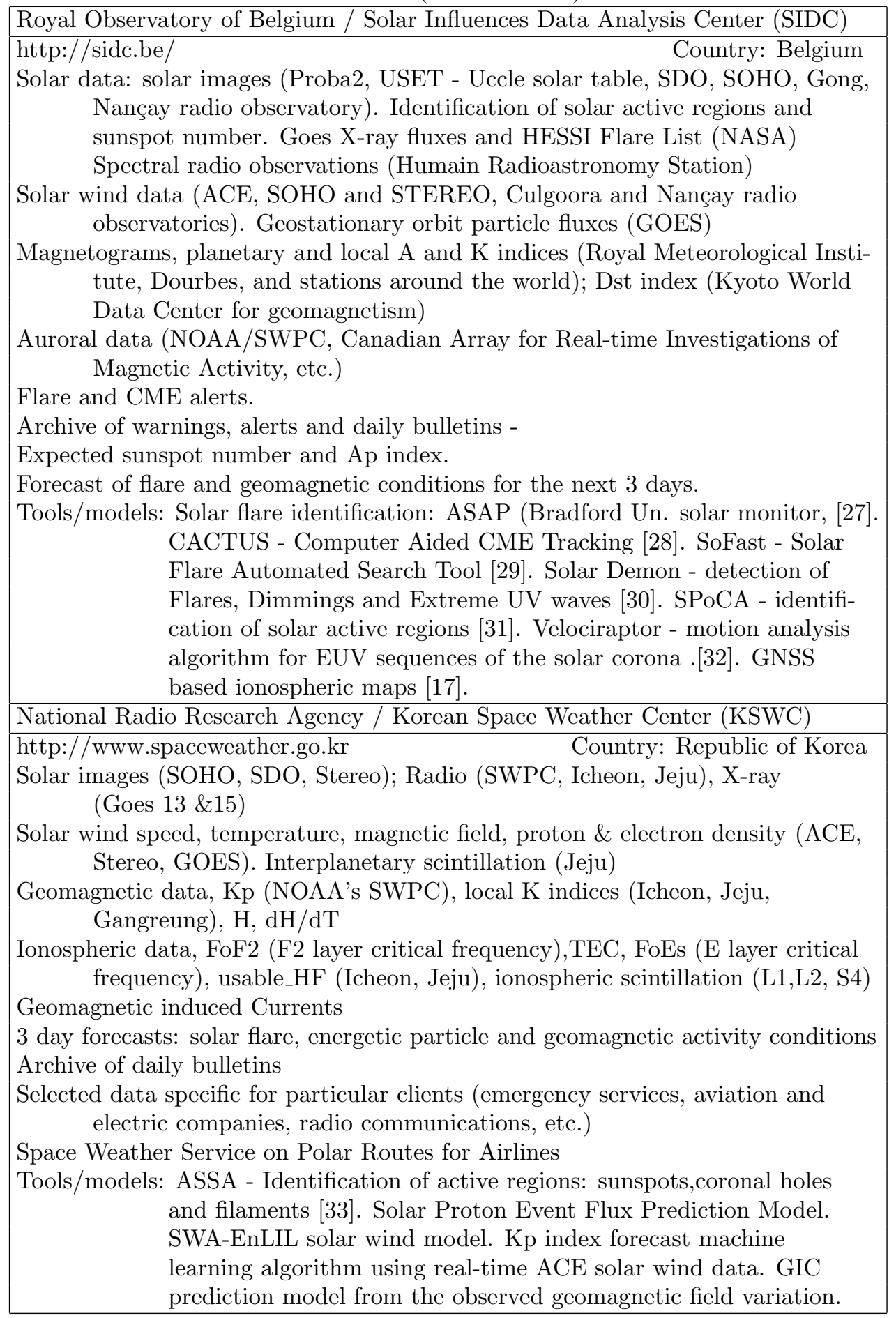


Table 1: (continuation)

National Institute of Information and Communications Technology (NICT) URL: http://swc.nict.go.jp/contents/index_e.ph Country: Japan Solar data: Solar images (Hinode, SOHO, SDO), sunspot number (SIDC) and F10.7 cm flux (NOAA/SWPC)

Solar wind parameters (ACE)

Energetic particle fluxes at geostationary orbit (GOES 13 \& 15)

Dst and AE indices

Monitoring and daily forecast of flare, energetic particle and geomagnetic activity levels

Ionospheric data Radio communications: E and F2 layers critical frequencies (Short Wave radio fadeout) world maps

3 day forecasts of flare, energetic particle and geomagnetic activity

Tools/models: Dst forecast by neural network.

Institute of Atmospheric Physics (IAP)

URL: http://rwcprague.ufa.cas.cz/ Country: Czech Republic

$\mathrm{H}$-alpha and white light solar observations (Ondrejov observatory).

Sunspot number X-ray (GOES) and 10.7 radio fluxes (Penticton).

Ionograms (Pruhonice observatory)

weekly geomagnetic activity report and forecast (Budkov geomagnetic observatory)

Archive of daily bulletins with description of past and future (2 day) solar activity conditions (flares and energetic protons)

Estudo e Monitoramento Brasileiro do Clima Espacial (EMBRACE)

URL: http://www.inpe.br/climaespacial/ Country: Brasil

Solar wind parameters (ACE)

Energetic particle fluxes at geostationary orbit (GOES 13 \& 15)

1000-2500 MHz solar radio flux (Brazilian Solar Spectroscope

Geomagnetic data: H, dH/dt (from stations across South America), AE, Dst and $\mathrm{Kp}$ indices

Ionospheric data: ionograms and ionospheric parameters (São Luis station)

Regional TEC and scintillation maps (GPS stations across the country)

Archive of daily bulletins with description of past and expected conditions in the last/next 24 hours

Models/tools: SUPIM-INPE/Sheffield University Plasmasphere-Ionosphere

model [34, 35]

Canadian Space Weather Forecasts Centre (CSWF)

http://www.spaceweather.gc.ca/ Country: Canada

Electron flux at geosynchronous orbit (GOES)

$10.7 \mathrm{~cm}$ solar radio flux (Dominion Radio Astrophysical Observatory. Penticton)

Planetary (GFZ, Potsdam) and local K indices (Ottawa, Meanook \& Victoria observatories)

Magnetograms from stations across the Canadian territory

Models/tools: Energetic electron flux [36]. Short (Wing-Kp) and long term geomagnetic activity forecast 
Table 1: (continuation)

\begin{tabular}{|l|}
\hline Servicio Nacional de Meteorologa Espacial (SeNMEs) \\
\hline URL:http://www.senmes.es/index.php \\
Solar observations (SOHO, SDO) \\
X-rays and solar energetic particles (GOES 13 \& 15$)$ \\
Solar wind data (ACE) \\
LDiñ Local Disturbance index for Spain \\
TEC map for the Iberian peninsula (Madrid Complutense University) \\
Flare, energetic particle fluxes at geostationary orbits, geomagnetic and induced \\
$\quad$ current monitoring \\
Daily bulletins with description of past and expected conditions \\
Tools/models: Severe geomagnetic activity warning [37] \\
Magnetosphere recovery time [38] \\
\hline
\end{tabular}

\subsection{Expertise in Automatic Warning}

Data acquisition, storage and their dissemination constitute part of the SW information systems. Computational infrastructures and human resources play a key role in the interpretation of this data. Daily and weekly bulletins of past and future conditions are issued after each institution's team of experts evaluate previous conditions and the forecasts produced by their computational tools. More information about some forecast tools will be presented in Section 4, but for now can be stated that many these are based on machine learning algorithms that take into account the large repositories of SW data currently available and information arriving in near real-time. An extensive review of modelling anf forecast tools can be found in the work of Tsagouri et al. [39]. These reports are made available to the general population by means of web portals and email.

The forecasting ability of some these automatic tools allows them to predict the occurrence of SW events in the near future as they evaluate the near past conditions and receive new real-time data. For instance, the wing Kp model [21] makes 1 and 4 hours in advance forecasts of geomagnetic activity level, by taking into account solar wind data. Other automatic algorithms were developed in order to identify SW events as they take place. CACTUS detects coronal mass ejections in LASCO image sequences [28] while SoFAST automatically detects EUV flares from SWAP data [29]. When such events or imminent events are identified some of these tools automatically generate an alert, which they distribute to the general population not only by email and the SW service's web page but also through Short Message Services (SMS). These alerts can also be made available through social media. The bulletins issued by these instruments are also stored for further analysis. In this manner some SW services have a system of automatic warnings to alert for the occurrence or imminent occurrence of SW events such as flares CMEs and geomagnetic storms.

NOAA/SWPC's web portal contains a graphical representation of the automatic SW alerts issued by them in the last seven days (www.swpc.noaa. gov/products/notifications-timeline). Similarly, the COMESEP alert system (www.comesep.eu/alert/), which consists of a network of alert tools (including among others CACTUS, SolarDemon and SEPforecast), has an user interface that allows the visualisation of its alerts.

Social media, web portals and applications constitute the most recognizable component of SW information systems. Web portals provide to the public the aforementioned observations, models, forecasts, bulletins and alerts. Additionally, they frequently include news and/or outreach sections with the purpose of increasing the general public's awareness to the importance of SW. Likewise, some web pages have sections specially dedicated to particular human activities, in which the information provided is custom tailored to the user's needs. Power grids are informed about the occurrence of CMEs, solar wind and geomagnetic parameters, are provided with GIC maps and, for countries at high latitudes, are provided with auroral models.

Satellite operators require information about geomagnetic and energetic particle activity, including the fluxes of high energy protons and electrons.

On the other hand, in addition to geomagnetic and flare activity indicators, users that rely on Global Positioning Systems (GPS) are also informed addition to a more generic web portal in which the afore- 
mentioned data is presented, out ionospheric parameters, particularly TEC and ionospheric scintillation maps.

Likewise radio communications require geomagnetic and flare activity indicators, ionospheric parameters (including ionospheric absorption maps) and again, auroral maps in the case of regions located at higher latitudes.

Finally in addition to the same data important for navigation and radio communications operators, aviation companies also require information about energetic particle fluxes.

In summary, not all institutions provide the same information, depending on the research interests of each institution and the aforementioned regional relevance of some SW phenomena. For instance, while CSWFC presents a monitoring of the geomagnetic field and forecasts concerning the occurrence of auroras, EMBRACE gives particular interest to the monitoring of the ionospheric conditions and the forecast of solar flares that could be responsible for radio communication blackouts at lower latitudes. Nonetheless, while many institutions focus on the regional impact of SW events, a few ones also focus in the global impact of SW providing models, observations, warnings and alarms that are used by the remaining institutions. A particular highlight must be given to NOAA's SWPC which, within the ISES organisation, acts as a central information hub between all members. Indeed, NOAA's SWPC is the source of data from telescopes such as GOES, ACE and STEREO.

\section{Activity indices}

Many SW monitoring institutions use a set of scales to characterise the degree of SW activity. These activity indices are mainly focused around three phenomena: the occurrence of flares, the influx of solar energetic particles and the strength of geomagnetic disturbances. For a matter of simplification these activity scales will be represented by the same lettering system used by NOAA's SWPC, respectively, the R, S and $\mathrm{G}$ scales. The emphasis on these phenomena results from their impact on multiple fields. For instance, solar flares can degrade high frequency communications and GPS signals, solar energetic particles (SEP) can have biological implications and affect satellite components, while geomagnetic storms can affect aviation and power grids.

The Spanish SeNMEs SW service from Alcalá de Henares University has a fourth activity scale (C) for the monitorisation of GICs. This is based on a Local Disturbance index computed for the Spanish territory (based on data from the San Pablo - Toledo geomagnetic observatory) and its temporal derivative. Other activity levels can be found, including the $\mathrm{H}$-scale for proton fluxes above $100 \mathrm{MeV}$ [40] or the radiation field activity index (D) for aviation altitudes of [41].

It is important to notice that some institutions use their own designations for each one of the aforementioned activity indices: NOAA/SWPC's "radio blackout" index (R) is analogue to NICT's "flare activity" level (phenomena which can cause radio blackouts). Likewise SWPC's radiation storm scale is analogue to CSWFC's Geostationary Satellite Environment scale. Additionally, the nomenclature used for each activity level can change from institution to institution. In this manner, what NICT defines as an "Active" Sun in terms of flare occurrence, corresponds to the occurrence of "Minor" and "Moderate" solar storms according to SWPC. Also while in some reports of SWS the lowest degrees of flare activity are classified as "Very Low" and "Low" instead of "Quiet" and "Eruptive" as it should be expected from the definition presented in SWS's web page.

On the other hand, despite the fact that the scales used by several organisations are based in the sane phenomena, not always they are defined using the same quantities. As an example, geomagnetic activity can be defined in terms of the Dst index, which is computed from data obtained at equatorial latitudes, or from geomagnetic indices such as the $\mathrm{Kp}$, Ap indices that are derived from instruments located at intermediate latitudes. Moreover, even when scales are based on the exactly same phenomena (e.g. the intensity of solar flares) different services can have different criteria. For instance, SWPC's R index is based on the occurrence of a single flare of a given intensity (e.g. a M5 flare is required for a "moderate" radio blackout), while Australia's SWS analogue index is based on the occurrence of a given number of flares of a given energy level (e.g. 1 to $4 \mathrm{M}$ class flares for a "moderate" solar flare activity). Tables 2 to 4 present, respectively, 
the criteria followed by several institutions for their flare, energetic particle fluxes and geomagnetic activity levels. Likewise Figure 1 shows a visualisation of the differences between the flare and geomagnetic activity levels.

Table 2: Comparison between the flare activity levels defined by NOAA, NICT and SWS.

\begin{tabular}{|c|c|c|c|}
\hline \multicolumn{2}{|c|}{ NOAA/SWPC } & NICT & SWS \\
\hline $\begin{array}{l}\text { Activity } \\
\text { level }\end{array}$ & $\begin{array}{l}\text { Flare } \\
\text { intensity }\end{array}$ & $\begin{array}{cc}\text { Activity } & \text { Flare } \\
\text { level } & \text { intensity }\end{array}$ & \begin{tabular}{|cc}
$\begin{array}{c}\text { Activity } \\
\text { level }\end{array}$ & Flare \\
& intensity
\end{tabular} \\
\hline None (R0) & A to $\mathrm{C}$ & Quiet $\mathrm{A}$ or $\mathrm{B}$ & Very low Weaker than $\mathrm{C}$ \\
\hline Minor (R1) & M1 & Eruptive C & C-class \\
\hline Moderate (R2) & M5 & Active $\mathrm{M}$ & Moderate 1 to 4 M-class \\
\hline Strong (R3) & $\mathrm{X} 1$ & Major & High $\quad 5$ or more M-class \\
\hline Severe (R4) & $\mathrm{X} 10$ & flares X & M5 or greater strength \\
\hline Extreme (R5) & $\mathrm{X} 20$ & & $\begin{array}{r}\text { Very High } 5 \text { or more M5 or } \\
\text { greater strength }\end{array}$ \\
\hline $\begin{array}{l}\text { Also } \\
\text { used by }\end{array}$ & $\begin{array}{l}\text { KSWC } \\
\text { SeNMEs }\end{array}$ & $\begin{array}{ll}\text { Also } & \text { SIDC } \\
\text { used by } & \end{array}$ & $\begin{array}{l}\text { Also } \\
\text { used by }\end{array}$ \\
\hline
\end{tabular}

Table 3: Energetic particle flux levels used by NOAA/SWPC and CSWFC.

\begin{tabular}{|c|c|c|}
\hline \multicolumn{2}{|c|}{ NOAA/SWPC } & CSWFC \\
\hline \begin{tabular}{|c|} 
Activity \\
level
\end{tabular} & $\begin{array}{l}\text { proton }(\geq 10 \mathrm{MeV}) \\
\text { flux } \\
\left(\text { prot. } \mathrm{cm}^{-2} \mathrm{sr}^{-1} \text { day }^{-1}\right)\end{array}$ & \begin{tabular}{|ll}
$\begin{array}{c}\text { Activity } \\
\text { level }\end{array}$ & electron flux \\
& (elect. $\mathrm{cm}^{-2} \mathrm{sr}^{-1} \mathrm{day}^{-1}$ )
\end{tabular} \\
\hline None (S0) & $\leq 10$ & \\
\hline Minor (S1) & $\overline{10}$ & Quiet \\
\hline Moderate (S2) & $10^{2}$ & Normal \\
\hline Strong (S3) & $10^{3}$ & Moderate $\geq 5 \times 10^{7}$ \\
\hline Severe (S4) & $10^{4}$ & $>5 \times 10^{8}$ \\
\hline Extreme (S5) & $10^{5}$ & Very high $\geq 5 \times 10^{9}$ \\
\hline \begin{tabular}{|l} 
Also \\
used by
\end{tabular} & $\begin{array}{l}\text { KSWC } \\
\text { SeNMEs }\end{array}$ & \\
\hline
\end{tabular}

Table 4: Comparison between the geomagnetic storm levels defined by SWPC, CSWFC, NICT and SWS space weather services.

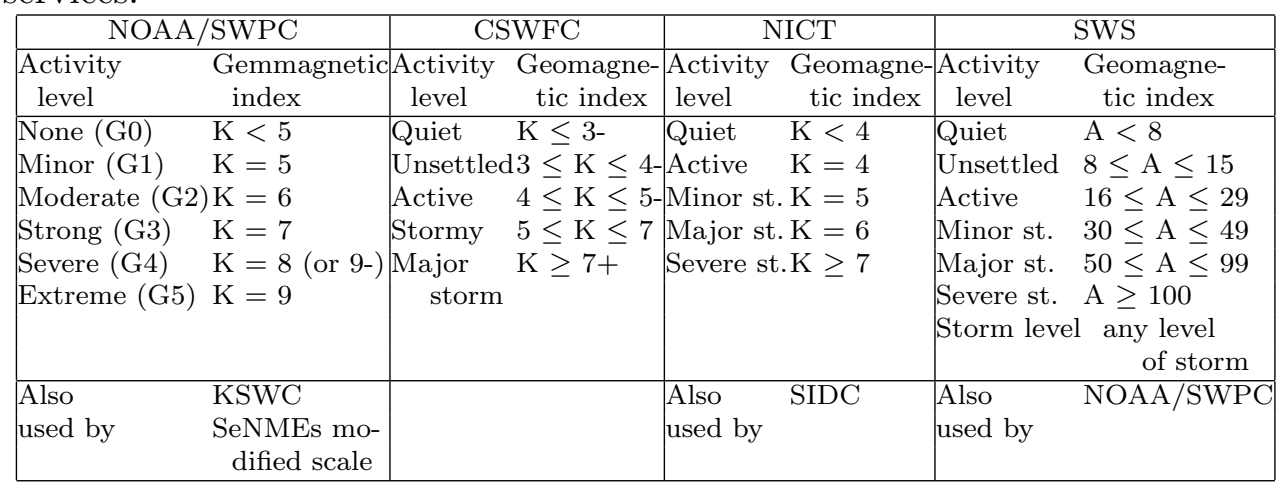

From Figure 1 we notice that several institutions subdivide NOAA/ SWPC's lowest flare and geomagnetic activity levels into several sub-catego- ries. This is particularly true in the case of CSWFC, since one of their main interests is the occurrence of auroras, whose minimum latitude at which they are visible strongly depends on the $\mathrm{Kp}$ index. For instance, for $\mathrm{Kp}=1$ auroras are visible at Fairbanks (Alaska) while for $\mathrm{Kp}=3$ they became visible as south as Calgary (Canada). Conversely, some scales group together NOAA's "Minor" to "Moderate" and the "Strong" to "Extreme" radio blackout levels. This grouping also makes sense taking into account that these levels are associated with the occurrence of $\mathrm{M}$ and X class flares respectively.

As a consequence of these definition differences, even if two institutions classify the activity degree of a given day taking into account the same observations, their classifications may be seen as divergent despite the fact that both classifications are correct according to their own scale system. Tables 5 to 7 illustrate this issue by comparing the classification, according to different scales, of the maximum flare and geomagnetic 


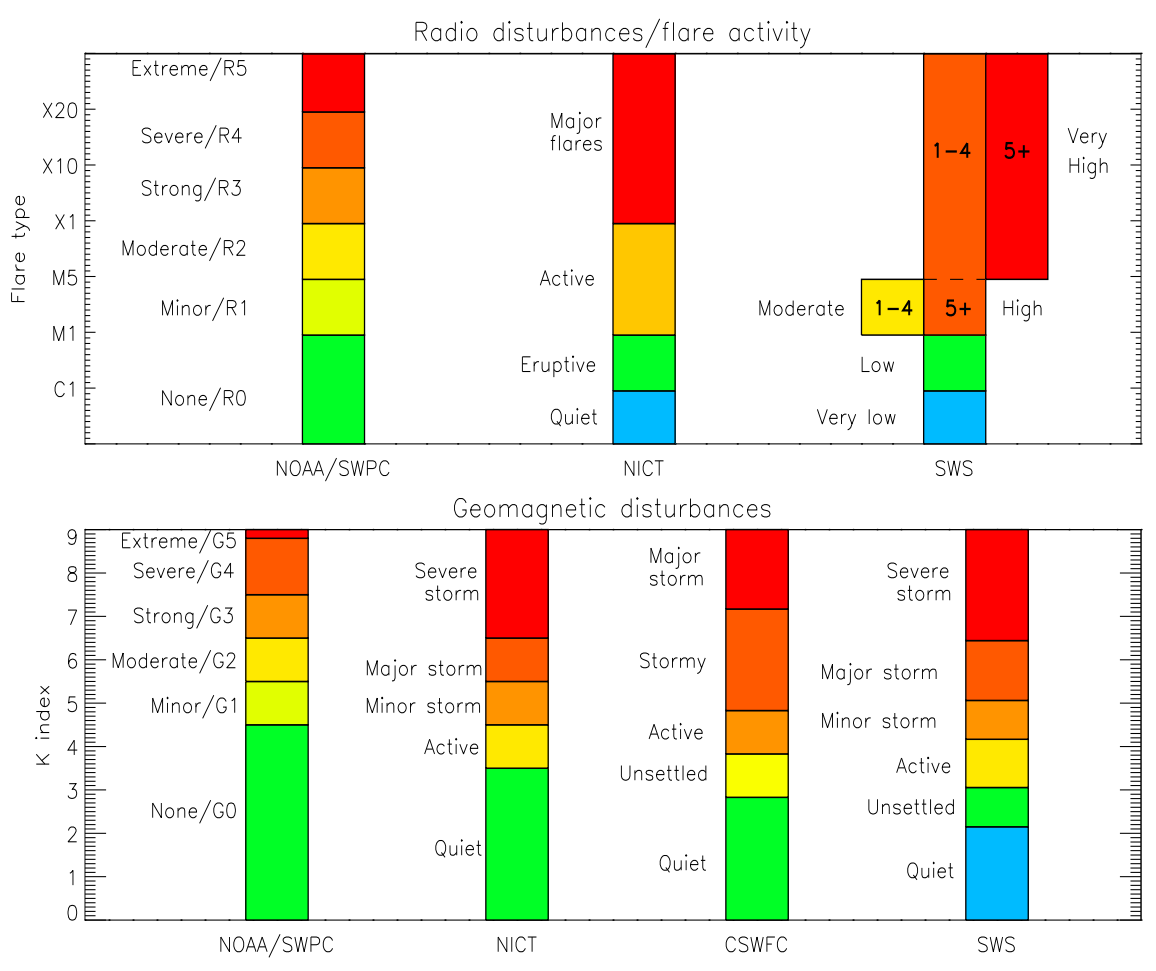

Figure 1: Top: comparison between the flare activity/radio disturbance levels used by NOAA/SWPC, NICT and SWS. Bottom: comparison between the geomagnetic activity levels used by NOAA/SWPC, NICT, CSWFC and SWS.

activity levels for each day of 2015. This classification was based on data from the Hinode Flare Catalogue (http://st4a.stelab.nagoya-u.ac.jp/hinode_flare/) of [19] and geomagnetic indices provided by the HelmholtzZentrum Potsdam (GFZ-Potsdam) World Data Center (ftp://ftp.gfz-potsdam.de/pub/home/obs/kp-ap/wdc/). Examples of this disparity of classifications can be found in the "Minor" (according NOAA/SWPC's scale) geomagnetic storms of March $12^{\text {th }}$ and September $29^{\text {th }} 2015$, which were classified as "High" activity in SWS's scale. Another possible scenario (which did not happened in 2015) is the occurrence of six M5 class flares on the same day. This would correspond to a "Moderate" activity according to NOAA's scale and to a "Very high" activity according to the scale of SWS.

Notice that in a few cases, particularly when the SW conditions are near the boundaries between activity levels, we can find some inconsistencies between the reported SW conditions and the daily activity classifications. For instance, SWS's bulletin of the $29^{\text {th }}$ of January of 2015 indicates a "moderate" flare activity in the previous day with only a C9.0 flare reported, yet according to their own scale system such flare corresponds to a "low flare activity".

Table 5: Comparison between the daily flare activity of 2005 (reported in the Hinode Flare Catalog) according to the radio blackouts/flare activity scales of NOAA/SWPC, SWS and NICT.

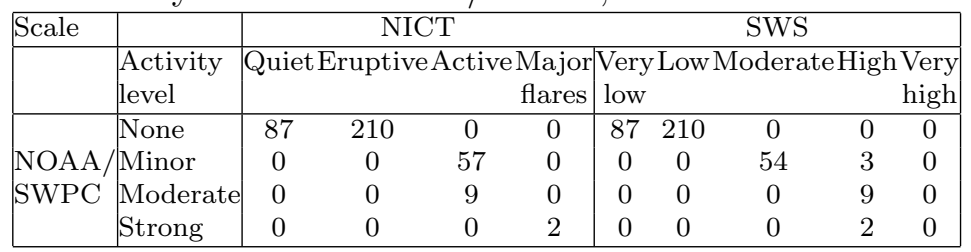

\section{Forecast of Space Weather conditions}

Several institutions have created their own forecast tools for the short term (hours to a couple of days) 
Table 6: Comparison of 2015's daily maximum geomagnetic activity (reported by GFZ-Potsdam's WDC) according to the scales of NOAA/SWPC, CSWFC, SIDC and SWS.

\begin{tabular}{|c|c|c|c|c|c|c|c|c|c|c|c|c|c|c|c|c|c|}
\hline \multirow[t]{2}{*}{\begin{tabular}{|l|} 
Scale \\
\end{tabular}} & \multirow[b]{2}{*}{$\begin{array}{c}\text { Activ. } \\
\text { level }\end{array}$} & \multicolumn{5}{|c|}{ CSWFC } & \multicolumn{5}{|c|}{\begin{tabular}{|c|} 
NICT \\
QuietAct.Min.Maj.Sev
\end{tabular}} & \multicolumn{6}{|c|}{ SWS } \\
\hline & & \multicolumn{5}{|c|}{$\begin{array}{r}\text { QuietUnset.Act.StormyMaj } \\
\text { st. }\end{array}$} & \multicolumn{5}{|c|}{$\begin{array}{r}\text { QuietAct.Min.Maj.Sev. } \\
\text { st. } \quad \text { st. } \quad \text { st. }\end{array}$} & \multicolumn{6}{|c|}{$\begin{array}{r}\text { QuietUnset.Act.Min.Maj.Sev } \\
\text { st. st. st. }\end{array}$} \\
\hline & None & 136 & 123 & 37 & 0 & 0 & 259 & 37 & 0 & 0 & 0 & 68 & 118 & 87 & 23 & 0 & 0 \\
\hline NOAA/ & Minor & 0 & 0 & 13 & 30 & 0 & 0 & 0 & 43 & 0 & 0 & 0 & 0 & 0 & 28 & 15 & 0 \\
\hline \multirow[t]{3}{*}{ SWPC } & Moder. & 0 & 0 & 0 & 18 & 0 & 0 & 0 & 0 & 14 & 4 & 0 & 0 & 0 & 0 & 18 & 0 \\
\hline & Strong & 0 & 0 & 0 & 5 & 0 & 0 & 0 & 0 & 0 & 5 & 0 & 0 & 0 & 0 & 0 & 5 \\
\hline & Extr. & 0 & 0 & 0 & 0 & 3 & 0 & 0 & 0 & 0 & 3 & 0 & 0 & 0 & 0 & 0 & 3 \\
\hline
\end{tabular}

Table 7: Comparison of 2015's daily naximum geomagnetic activity (reported by GFZ-Potsdam's WDC) in SWS's scale against the daily classifications using the scales of CSWFC and SIDC.

\begin{tabular}{|c|c|c|c|c|c|c|c|c|c|c|c|}
\hline \multirow[t]{2}{*}{ Scale } & & \multicolumn{5}{|c|}{ CSWFC } & \multicolumn{5}{|c|}{ NICT } \\
\hline & Activity & Quiet & nsett & Acti & torm & Major & Quie & Ictiv & Minc & Maj & evere \\
\hline \multirow{6}{*}{ SWS } & Quiet & 68 & 0 & 0 & 0 & 0 & 68 & 0 & 0 & 0 & 0 \\
\hline & Unsettled & 68 & 50 & 0 & 0 & 0 & 118 & 0 & 0 & 0 & 0 \\
\hline & Active & 0 & 73 & 14 & 0 & 0 & 73 & 14 & 0 & 0 & 0 \\
\hline & Minor storm & 0 & 0 & 36 & 15 & 0 & 0 & 23 & 28 & 0 & 0 \\
\hline & Major storm & 0 & 0 & 0 & 33 & 0 & 0 & 0 & 15 & 14 & 4 \\
\hline & Severe storm & 0 & 0 & 0 & 5 & 3 & 0 & 0 & 0 & 0 & 8 \\
\hline
\end{tabular}

prediction of SW conditions [39, for instance]. Flare activity forecast generally involves the use of machine learning algorithms which identify sunspot groups using magnetograms and continuum images of the Sun. The determination of the total magnetic flux in the active regions, or at least the classification the sunspot groups according to structure and complexity [42], is followed by the use statistical models which correlate these fluxes/classifications with recent flaring activity. Forecasts are generally provided under the form of probabilities of occurrence of $\mathrm{C}, \mathrm{M}$ and $\mathrm{X}$ type flares. Among other examples of such tools we find the Korean ASSA - Automated Solar Synoptic Analysis [33] and the British ASAP - Automated Solar Activity Prediction tool - ASAP [43, 27].

The identification/characterisation of coronal mass ejections with tools such as CACTUS [28] and Velociraptor [32], in combination with solar wind data and models such as the WSA-Enlil [14, 15] are fundamental to predict the occurrence of solar proton events. Solar flare information and near-Earth space environment data are used to predict the occurrence of solar radiation storms by tools such as: SEPForecast [44] UMASEP [45], NOAA/SWPC's Relativistic Electron Forecast Model [46] or KSWC's Solar Proton Event Flux Prediction Model. Other machine learning algorithms are used along with solar wind data and recent geomagnetic indices to forecast the future geomagnetic conditions. Among other we find the Wing-Kp tool [21], KSWC's machine learning Kp Prediction Model and the Hybrid Dourbes K model [47]. On the other hand, Devos et al. [13] propose a statistical tool for the arrival and geoeffectiveness forecast of CMEs. Finally, in addition to a computational tool for the identification of the start of geomagnetic storms [37], the Spanish SeNMEs SW service has a tool for the estimation of the magnetospheric recovery time after a storm [38].

A comparison between the forecasted and observed SW conditions allows validating these tools $[11,48,13$, for instance]. Indeed NOAA/SWPC's web portal has a section dedicated to the verification of the quality of their forecasts (http://www.swpc.noaa.gov/content/forecast-verification), while in NICT's web page can be found a comparison of the forecast accuracy of a few SW services (http://seg-web.nict.go.jp/cgibin/forecast/eng_forecast_score. cgi).

Forecasts along a full Carrington rotation (i.e. about 27 days) are also possible. Recurrence models assume that the active regions retain the same level of activity for an entire solar rotation [49]. In this manner one can forecast sunspot numbers, $10.7 \mathrm{~cm}$ solar radio fluxes and geomagnetic indices. These two properties, which are strongly dependent on the solar cycle, can also be predicted over periods of weeks and months. For instance SWPC presents such predictions based on the results of the Solar Cycle 24 Prediction Panel (http://www.swpc.noaa.gov/products/solar-cycle-progression). Yet these estimates are only reliable well after a new solar cycle has begun, i.e. about 3 years after the minimum sunspot number is achieved 
$[50]$.

\section{Daily forecast comparison}

Not only forecasts are derived using different tools, but the way these predictions are presented is not the same for every institution. Some SW services indicate the expected geomagnetic indices or flare occurrence probabilities, while others provide activity levels. Moreover, the range of possible scenarios predicted by each institution is not always the same: due to their rarity, most SW services only indicate two possible scenarios in respect to the occurrence of solar energetic p article storms of any intensity ("quiet" or "event in progress/expected"), while the two probabilities of energetic particles storm provided by KSWC (one for "minor to moderate" storms and the other for events of higher intensity) result in three possible situations. Additionally, as seen in Tables 2 to 4, not all institutions use the same activity scales.

Despite the differences in the nomenclature and methods used by several SW services it is possible to compare some of these forecasts. Geomagnetic indices can be converted into geomagnetic activity levels. Likewise, the probabilities of occurrence of $\mathrm{C}, \mathrm{M}$ and $\mathrm{X}$ flare events can be used to compute flare activity levels. In this manner, a X type flare probability above $50 \%$ is similar to forecasting the occurrence of "strong" to "extreme" events according to NOAA/SWPC's scale. Even when there is no correspondence between two forecasts one can at least compare the accuracy of the SW services that issued them, i.e. their ability to predict the correct conditions. Indeed, the Japanese space weather service has a tool for evaluating the accuracy of the forecasts issued by NICT KSWC, SWPC, SWS, SIDC and the Chinese SW service SAPC. This tool can be found at: http://seg-web.nict.go.jp/cgi-bin/forecast/eng_forecast_score.cgi. However these comparisons, which comprise mostly institutions from the Asia-Pacific region, do not include forecasts concerning the occurrence of solar energetic particle (SEP) events.

The following paragraphs contain an extension of NICT's analysis, taking into account additional SW warning centers and including an evaluation of their ability to predict solar proton storms. The biggest limitation to our study results from the fact that we could only evaluate SW services which have available an archive of previous reports. In this manner, we could not take into account the forecasts of NICT and SAPC, but we were able to analyse the predictions of EMBRACE and IAP. Nevertheless the availability of data from IAP and, especially EMBRACE, limited our analysis from the beginning of 2015 onwards. Table 8 provides a brief description of the different parameters predicted by each one of these institutions. For a matter of consistency, geomagnetic indices and flare probability forecasts were converted into NOAA/SWPC and SWS's activity scales. The forecasts of SW conditions were obtained from the "daily" bulletins issued by the aforementioned SW services (EMBRACE only publishes them on workdays). While many reports include predictions for the next 24, 48 and 72 hours, in the case of EMBRACE and IAP forecasts are only issued for the next 24 hours. For this reason we only took into account the one day in advance forecasts.

Our evaluation of the accuracy of the aforementioned SW services consisted in determine the percentage of forecasts in which was predicted the correct activity level. This activity level was defined according to NOAA/ SWPC's or SWS scale, depending on the properties forecasted by each institution. These daily activity levels were computed taking into account the Hinode Flare Catalogue [19], the "5-minute GOES-13 Solar Particle and Electron Flux" provided by NOAA/SWPC (ftp://ftp.swpc.noaa.gov/pub/ lists/particle/) and the geomagnetic indices of the GFZ-Potsdam WDC.

\subsection{Flare forecast}

As seen in Table 8, SWPC, KSWC, EMBRACE and IAP provide the probability of occurrence of M and $\mathrm{X}$ type flares. These values can be compared against each other. Figure 2 shows that the forecasts of these institutions follow similar trends: The variations of the forecasted flare probabilities behave in a similar manner as the variations of the flare activity expected from Hinode Flare Catalogue (Fig. 2's lower panel). In this figure are also noticeable the gaps resulting from the fact that EMBRACE only issues reports on workdays.

These probabilities of flare occurrence can be used to compute the level of radio blackouts expected according to NOAA's scale. Following SIDC's procedure, we consider that a given activity level is forecasted whenever a flare probability above a certain threshold (in their case $50 \%$ ). In this way, we consider that 
Table 8: Data forecasted by SWPC, SWS, SIDC, KSWC, EMBRACE and IAP.

\begin{tabular}{|c|c|c|c|c|c|}
\hline SWPC & KSWPC & SIDC & SWS & IAP & EMBRACE \\
\hline $\begin{array}{l}\text { Probabilities } \\
\text { of } \mathrm{M} \text { and } \mathrm{X} \text { flares }\end{array}$ & $\begin{array}{l}\text { Probabilities: } \\
1 \leq R_{N O A A} \leq 2 \\
3 \leq R_{N O A A} \leq 5\end{array}$ & $\begin{array}{l}\text { Type of flares } \\
\text { expected }\end{array}$ & $\begin{array}{l}\text { Possible } \\
\mathrm{R}_{S W S} \text { range }\end{array}$ & \begin{tabular}{|} 
Probability \\
of $\mathrm{C}, \mathrm{M}$ and \\
$\mathrm{X}$ flares \\
$\mathrm{R}_{S W S}$ range
\end{tabular} & \begin{tabular}{|} 
Probability \\
of $\mathrm{M}$ and \\
X flares
\end{tabular} \\
\hline $\begin{array}{l}\text { Probability } \\
\text { of proton storm } \\
\text { (any level) }\end{array}$ & $\begin{array}{l}\text { Probabilities: } \\
1 \leq S_{N O A A} \leq 2 \\
3 \leq S_{N O A A} \leq 5\end{array}$ & $\begin{array}{l}\text { Proton events: } \\
\text { quiet or } \\
\text { in progress/expected }\end{array}$ & & $\begin{array}{l}\text { Probability of } \\
\text { proton events } \\
\text { (any level) }\end{array}$ & \\
\hline $\begin{array}{l}\mathrm{G}_{S W S} \text { range }^{a} \\
\text { expected } \mathrm{K} \text {-index }\end{array}$ & $\begin{array}{l}\text { Probabilities: } \\
1 \leq G_{N O A A} \leq 2 \\
3 \leq G_{N O A A} \leq 5\end{array}$ & $\begin{array}{l}\mathrm{G}_{S I D C} \text { expected } \\
\text { value }\end{array}$ & $\begin{array}{l}\text { Possible } \\
\mathrm{G}_{S W S} \text { range }\end{array}$ & & \\
\hline
\end{tabular}

"minor" to "moderate" flare activity is expected when the probability of M-flares occurrence is above $50 \%$, while "strong" to "extreme" events are expected when the probability X type flares is above this $50 \%$ threshold. On the other hand, the equivalence between NOAA/SWPC's radio blackout activity and SIDC's solar flare activity (seen in Figure 1) allows comparing SIDC's forecasts against those of SWPC, KSWC, EMBRACE and IAP. Figure 3 presents a comparison of these forecasts between the beginning of June and the middle of July of 2015, matching them against the daily flare activity classification derived from Hinode's flare catalogue [19]. Figure 3 and the histogram shown in Figure 4 indicate that these institutions are able to correctly predict the activity levels between $74 \%$ and $83 \%$ of the cases.

Nevertheless it is important to notice that they fail to forecast the $\mathrm{X}$ class flares took place on the $11^{\text {th }}$ of March and $5^{\text {th }}$ of May. Indeed, as in the case of Figure 2's central panel, the probability of X-ray flares is never above the $50 \%$ threshold. Given the small amount of X ray events available in our data we cannot make conclusive statements about the forecast abilities concerning more energetic events (for that we refer to each SW service validation studies).

The daily bulletins from IAP provide not only probabilities of flare occurrence, but also forecasts of the flare activity level expected according to SWS's scale. Thus the later predictions were compared against the daily SWS-scale flare activity classification and SWS's forecasts (Fig. 3's lower panel). As seen from the histogram on Figure 4, the SWS-scale forecasts of IAP and SWS have accuracies similar to the ones of the institutions evaluated before. Yet the slightly higher accuracy of IAP's forecasts is partially due to the fact that it tends to present a higher range of possible solutions (for instance they can go from "moderate" to "very high").

\subsection{Solar radiation storms}

In respect to the occurrence of solar radiation storms neither SWS nor EMBRACE include a forecast of this type of phenomena, while SDIC forecasts the occurrence of solar particle storms, indicating if the conditions are quiet or if an event is starting/continuing. On their own turn, SWPC, KWSC and IAP provide the probability of occurrence of any event above NOAA's "minor" level. Furthermore, since May of 2015 KSWC started to compute such probabilities for two types of events: those of "moderate" to "medium" intensity and events of higher intensity. The use of such wide range of possible scenarios makes sense taking into account the rarity of these events. Indeed it makes little sense to compute the probability of occurrence of "severe" events, which only take place about three times per solar cycle.

An in the case of the solar flares, we considered that an event probability above $50 \%$ corresponds to forecasting a solar radiation storm. These forecasts were compared against GOES $10 \mathrm{MeV}$ particle flux data available at: ftp://ftp.swpc.noaa.gov/pub/lists/particle/. Despite no information is provided prior to the $30^{\text {th }}$ of May of 2015, an inspection to SDIC and SWPC's bulletins indicated that no "minor" solar proton event took place between the beginning of 2015 and that date.

Figure 5 is an example of how the probabilities inferred by SWPC, KWSC and IAP seem to follow the evolution of the daily proton fluxes measured by the GOES 13 satellite.

From Table 9 we conclude that if we use these probabilities to predict the occurrence of solar radiation 


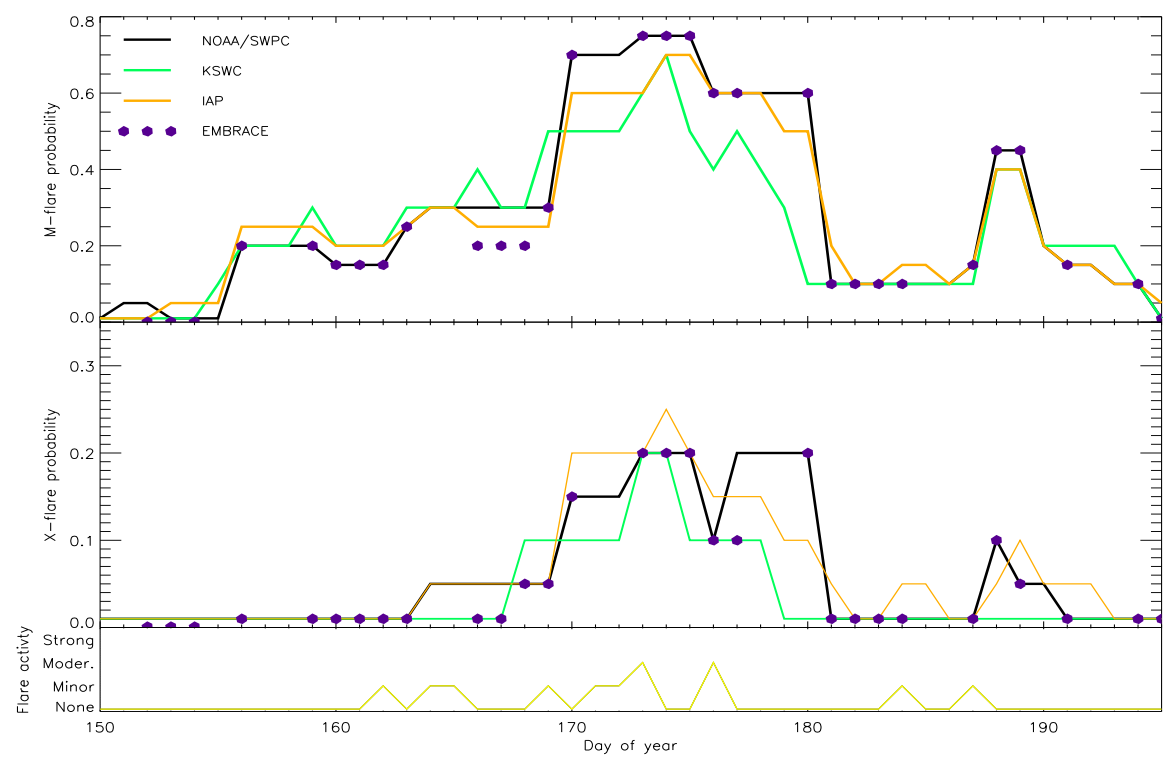

Figure 2: Forecast of the probability of occurrence of $\mathrm{M}$ (top panel) and $\mathrm{X}$ class flares (middle panel) according to SWPC (black lines), KSWC (green lines), IAP (orange lines) and EMBRACE (purple dots), from the beginning of June to the middle of July of 2015. Bottom: classification of the daily flare activity reported in the Hinode Flare Catalog according to NOAA/SWPC's radio blackout scale.

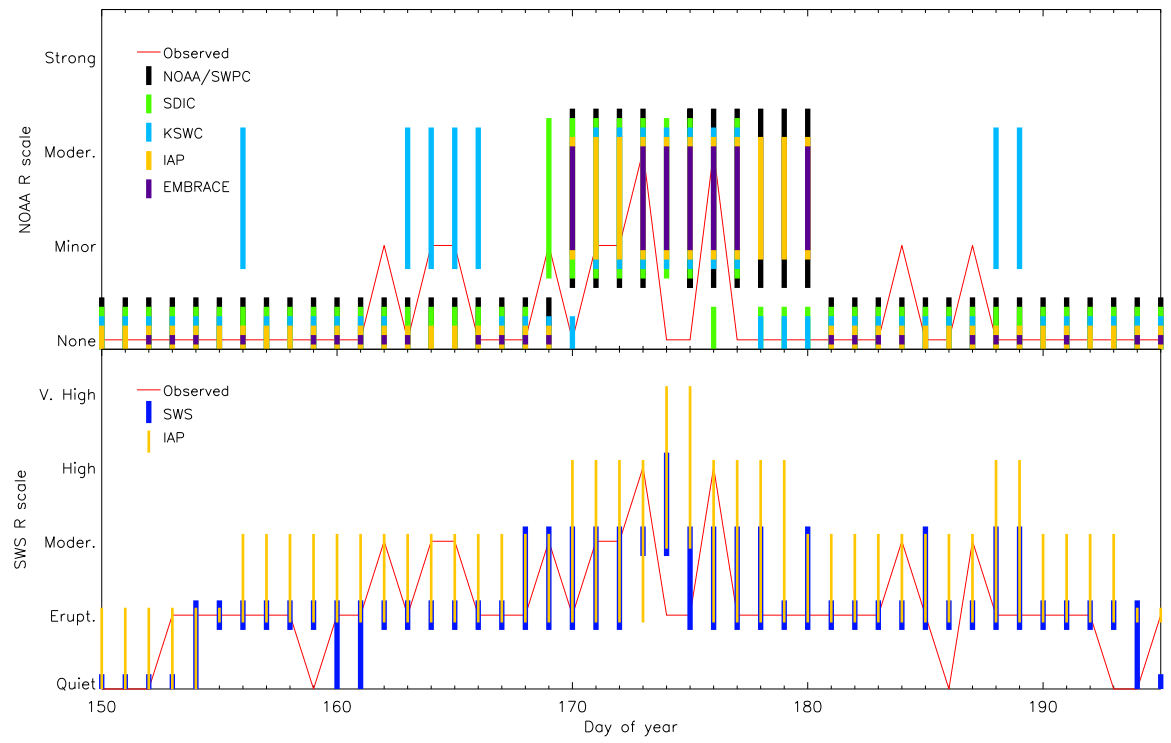

Figure 3: Top: Flare activity level (according to NOAA/SWPC's scale) expected from the flare probabilities forecasted by NOAA/SWPC (black), KSWC (cyan), IAP (orange), EMBRACE (purple) and the flare activity level forecasted by SIDC. Bottom: Flare activity levels (in SWS's scale) forecasted by SWS (thick blue bars) and IAP (thin yellow bars). The height each vertical bar corresponds to the uncertainty associated with each forecast, The red lines correspond to the daily flare activity from the beginning of June to the middle of July of 2015 . 


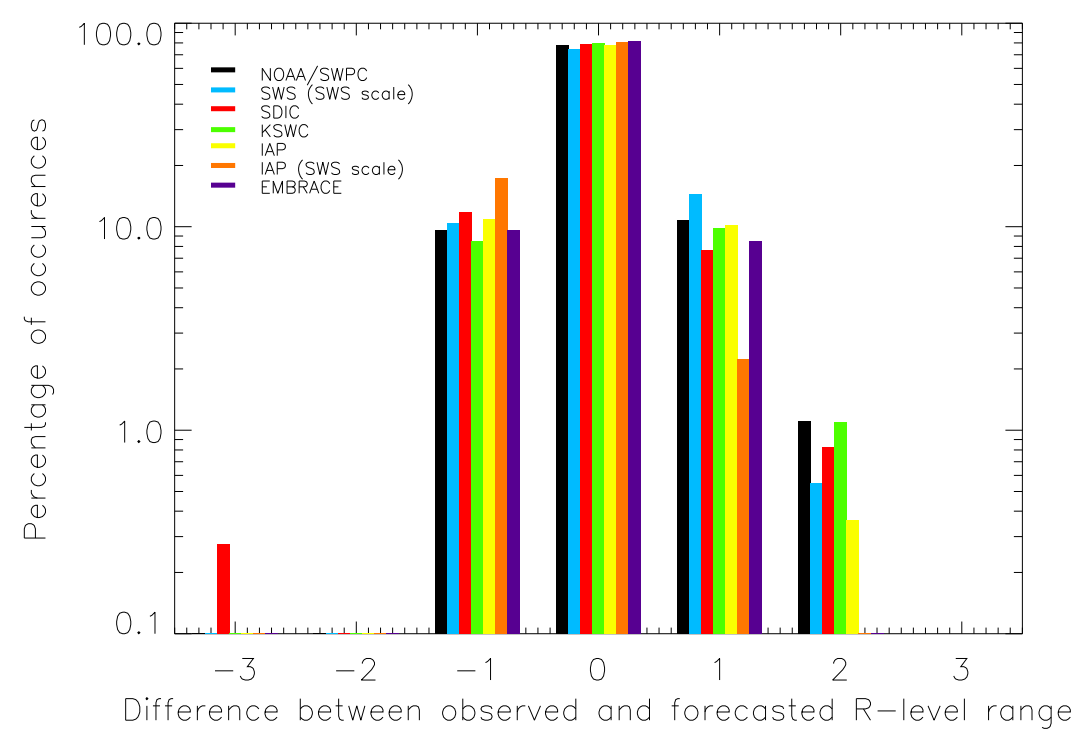

Figure 4: Histogram of the difference between the forecasts show in Figure 3 and the maximum daily flare activity (in the NOAA/SWPC and SWS scales) derived from the Hinode Flare Cataloge. The black, red, green, yellow and purple bars correspond, respectively, to the NOAA/SWPC scale forecasts of NOAA/SWPC, SDIC,KSWC, IAP and EMBRACE. The SWS scale forecasts of SWS and IAP are represented by the cyan and orange bars. Zero corresponds to daily conditions within the predicted intervals.

storms (assuming that an event will take place when the probability of occurrence is at least $50 \%$ ) we obtain results that do not differ that much from institution to institution.

Table 9: Comparison of the days in which, according to SWPC, KSWC and IAP, the probability of occurrence of solar radiation storms $\left(\mathrm{P}_{\text {event }}\right)$ is above or under $50 \%$ against the quiet or active days in which those solar radiation storms took place.

\begin{tabular}{|l|c|c|c|c|}
\hline & $\begin{array}{c}\text { SWPC/NOAA } \\
(363 \text { days })\end{array}$ & $\begin{array}{c}\text { SIDC } \\
(365 \text { days })\end{array}$ & $\begin{array}{c}\text { KSWC } \\
(365 \text { days })\end{array}$ & $\begin{array}{c}\text { IAP } \\
(274 \text { days })\end{array}$ \\
\hline $\mathrm{P}_{\text {event }}<50 \%$, quiet day & 96.4 & 84.7 & 97.3 & 96.0 \\
$\mathrm{P}_{\text {event }} \geq 50 \%$, quiet day & 1.1 & 11.8 & 0.3 & 0.4 \\
$\mathrm{P}_{\text {event }}<50 \%$, active day & 0.8 & 1.1 & 0.8 & 2.2 \\
$\mathrm{P}_{\text {event }} \geq 50 \%$ active day & 1.7 & 0.5 & 1.6 & 1.5 \\
\hline
\end{tabular}

\subsection{Geomagnetic activity}

SWPC provides two alternative geomagnetic forecasts: its "Report on Solar and Geomagnetic Activity" (RSGA) provides a qualitative description of the conditions expected according to SWS's scale, while its "3 day forecast" bulletin offers a breakdown of the K-indices expected. The maximum K index of that day can be used to infer the maximum geomagnetic activity level according to NOAA/SWPPC's scale. On the other hand, the similarities between the geomagnetic scales used by SDIC and NOAA/SWPC (Table 4) allow us to convert SDIC's geomagnetic forecasts into NOAA/SWPC's scale. Additionally, KSWC provides the probabilities for the occurrence of "minor to moderate" and higher intensity storms. As before, this allows defining a range of possible geomagnetic conditions (in our case assuming a 50\% critical threshold).

Figure 6's top panel presents a comparison of the geomagnetic NOAA/ SWPC scale forecasts of NOAA/SWPC, KSWC and SIDC, against the daily geomagnetic activity levels expected from the data of Potsdam's Helmholtz-Zentrum. In the same manner, Figure 6's lower panel also shows similarities between NOAA/SWPC and SWS geomagnetic forecasts provided in SWS's scale. Figure 7 shows that the accuracy of the four institutions evaluated here (i.e. their ability to predict the geomagnetic activity level for the next day) range between $68 \%$ and $89 \%$. 


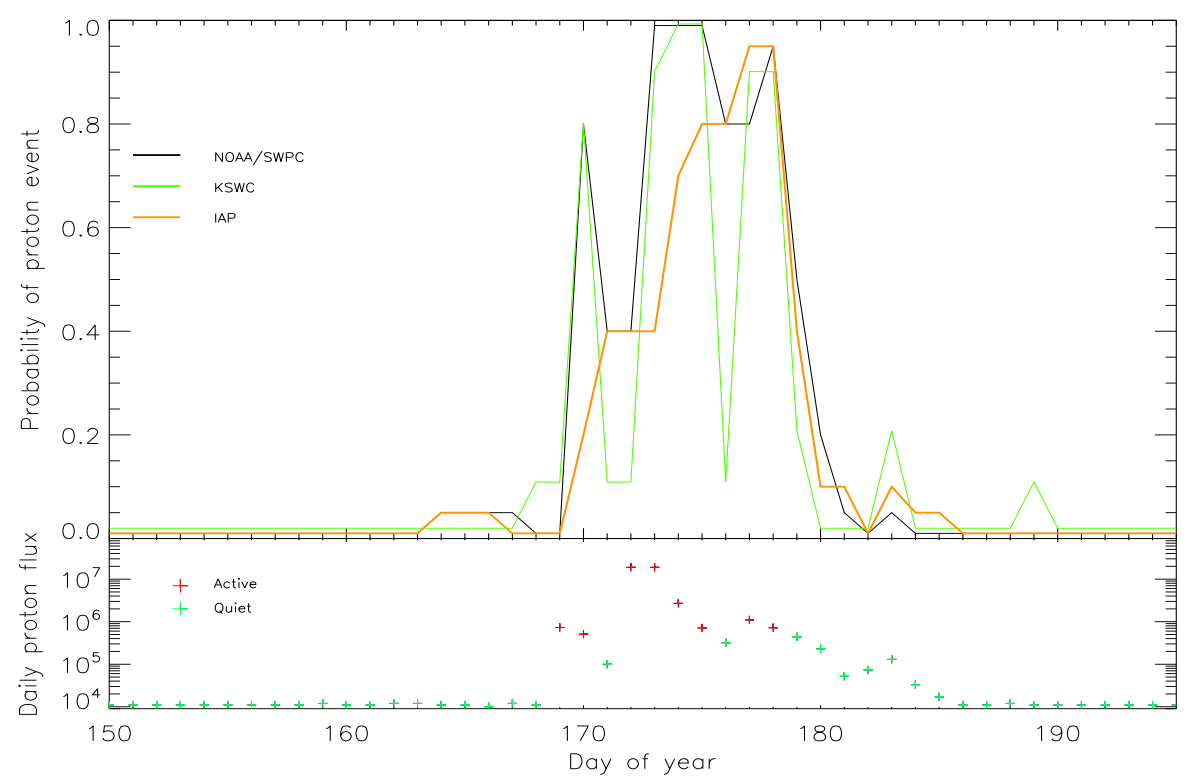

Figure 5: Top: Comparison of the probabilities of occurrence of energetic proton events according to SWPC (black) KSWC (green) and IAP (orange) from the beginning of June to the middle of July of 2015 Bottom: Daily solar proton fluxes $(>10 \mathrm{MeV})$ measured by the GOES-13 satellite. The red signs correspond to the days in which a solar radiation storm took place (i.e. at least one of GOES-13 5 minute measurements was at least 10 protons/cm2-s-sr), while the green signs correspond to quiet days.

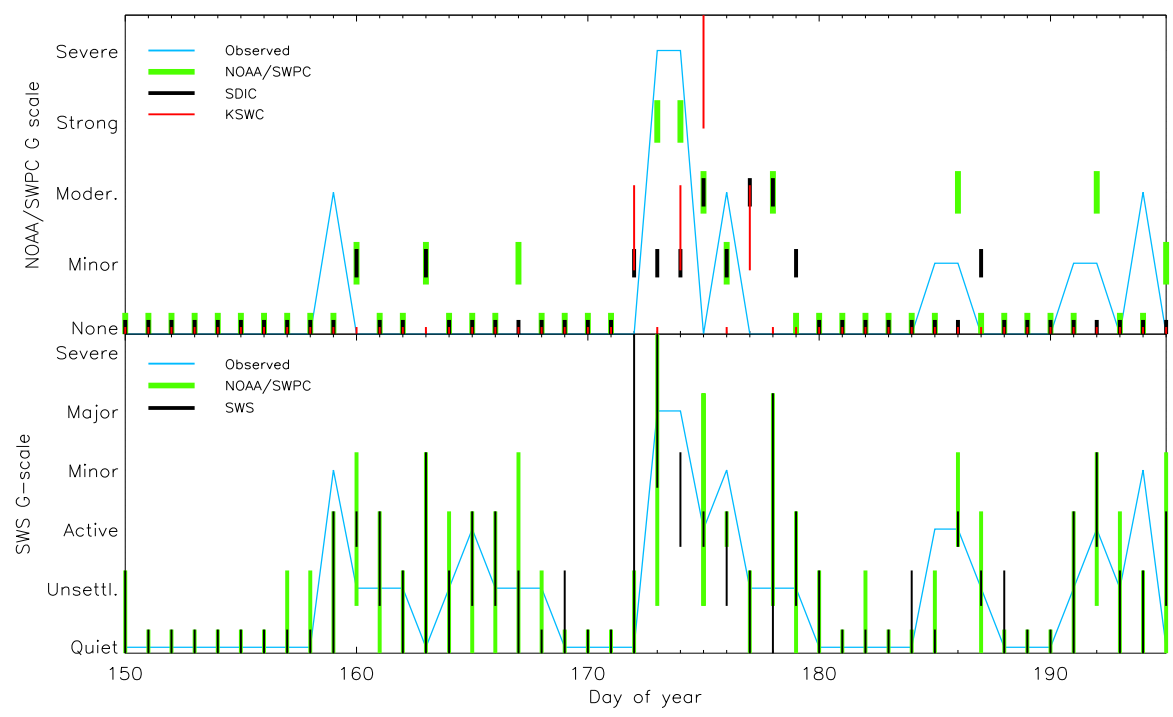

Figure 6: Top: Geomagnetic conditions forecasted by NOAA/SWPC (green) SIDC (black), KSWC (red) and NICT (orange), in NOAA/SWPC's scale, between the beginning of June and the middle of July of 2015. Bottom: Geomagnetic forecasts of NOAA/SWPC (green) and SWS (black), in SWS's scale, for the same time interval. The cyan lines correspond to the daily geomagnetic classifications according to the $\mathrm{K}$ and $\mathrm{A}$ indices provided by GFZ-Potsdam WDC. 


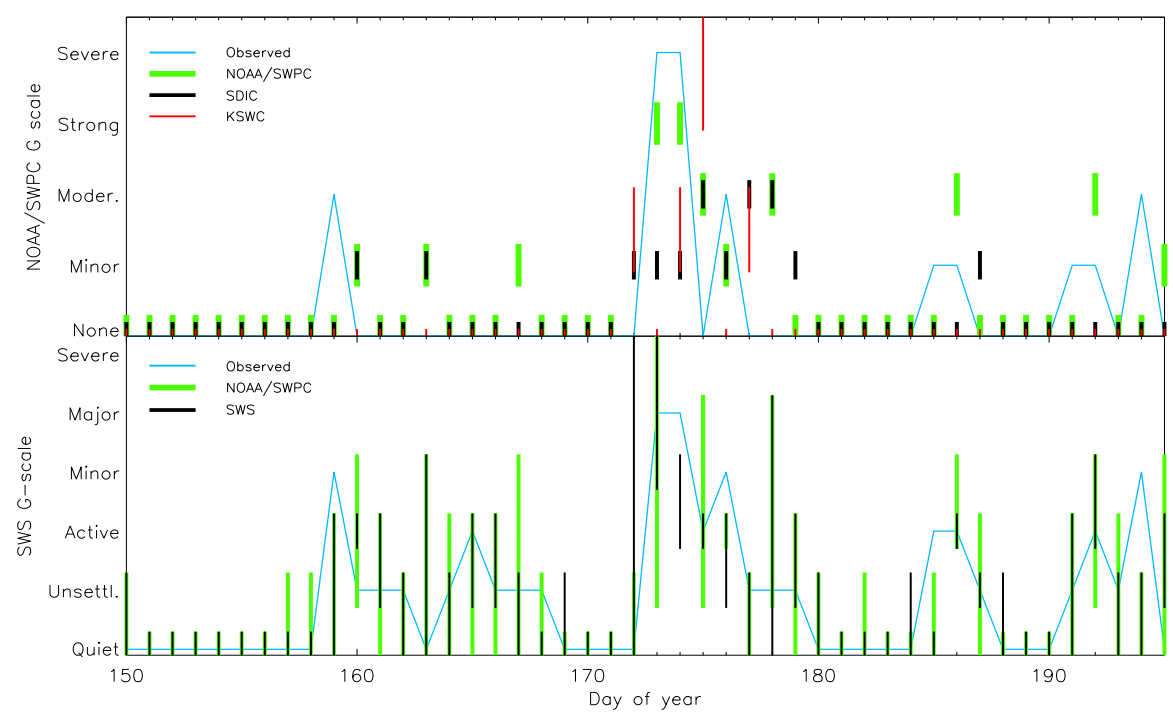

Figure 7: Histogram of the differences between the observed geomagnetic conditions and the forecasts for 2015 of NOAA/SWPC (dark and light blue respectively for the forecasts in NOAA/SWPC and SWS scales) the predictions of SWS's in its own scale (black), plus the NOAA/SWPC scale forecasts of SDIC (green) and KSWC (red).

\section{Conclusions}

From the analysis of different SW Information Systems across the globe we conclude that the information provided by them generally revolves around three topics. The first one concerns solar activity, including global activity indicators (sunspot number, $10.7 \mathrm{~cm}$ radio flux), plus the identification and characterisation of active regions (sunspots, filaments, coronal holes) and events (flares and CMEs). The second topic concerns the properties of the solar wind and near-Earth vicinity, while the last one involves geophysical properties, in particular geomagnetic and ionospheric characteristics (including radio propagation parameters). The variety and volume of data involved in the monitoring/forecast of SW conditions fall well within the Big Data paradigm. Additionally, it was noticed that several SW warning centers use scale sets in order to characterise different types of SW activity.

The research institutions associated with these SW services tend to focus their attention on SW phenomena within their scientific interests, and specially, on events that have a regional relevance on their geographic locations. Due to the aforementioned regional impact, SW services tend to use data of a regional/national origin. Nevertheless in the case of some global parameters (e.g. sunspot number, $10.7 \mathrm{~cm}$ solar flux. K and Dst indices) there are a few organisations and projects of reference worldwide, which act as a hub of information for that data. This is particularly true in the case of NOAA's SWPC.

Many institutions have developed their own strategies and tools for monitoring and forecasting the SW conditions. Some of these tools are able to alert for the occurrence of SW events (or their imminent occurrence) in an automatic way. Nevertheless the modelling of SW conditions still requires further developments to fully satisfy what we already know about Space Weather. This effort is currently under way which, simultaneously, is pushing furthermore our computational needs.

Our analysis of the type of information provided by SW Information Systems included other institutions besides those mentioned here. Among others, the South African National Space Agency (SANSA), Met Office Space Weather Operations Centre (MOSWOC), Lund Space Weather Center or the National Physical Laboratory of India. The conclusions that we arrived for these institutions were similar to the ones presented in this article.

From our evaluation of the activity level forecasts of six SW warning centers we conclude that these 
institutions have similar degrees of accuracy (generally around the order of 70 to $80 \%$ ). Yet our sample, comprised by the daily forecasts of 2015 , was clearly dominated by quiet days, which are the easiest to forecast. This puts some constrains in our conclusions.

We already expected that NOAA/SWPC, SWS, SIDC and KSWC had similar levels of accuracy in the forecast of flare and geomagnetic conditions from NICT's forecast comparison tool. Yet that conclusion has been expanded now to the forecasts of IAP and EMBRACE. Additionally, we evaluated the forecasts of solar particle storms, not taken into account by NICT's comparisons.

This study was limited by the amount of data available from the smallest institutions (particularly EMBRACE) and due to rarity of the highest energy events. Therefore it is important to increase the size of our sample in order to have a better sampling of the stormy conditions. Yet some time is required before the final calibration of SW conditions is made available. For instance, the final calibration of geomagnetic data of a given year is only obtained in the following year. On the other hand, as more institutions make available their archive of daily bulletins, we will be able to include them.

Without any doubt, the most important conclusion from this study is that the use of the different scales currently available for the classification/forecast of SW events can produce divergent results, despite the fact that these classifications/forecasts are correct according to their own scale.

Nowadays this has a particular relevance since, as more countries are starting to develop their own SW services, the need of homogenising their forecasts is becoming an even more urgent matter.

Acknowledgments. The authors would like to thank the diligent revision made by JCIS's Editor-in-Chief R. R. Rosa. FJGP thanks support provided through grant in scope of project "Implementação do SPINLab na componente dos dados solares" (UID/Multi/00611/2013) co-financed by Foundation for Science and Technology (FCT) and the program COMPETE 2020 Operational Programme Competitiveness and Internationalization (project: POCI-01-0145-FEDER-006922) The authors also acknowledge the data provided by the SW services evaluated here (NOAA/SWPC, Australian Bureau of Meteorology's SWS, SIDC, NICT, KSWC, AIP and EMBRACE) and the geomagnetic data from the Helmholtz-Zentrum Potsdam WDC. This work was also carried out using the Hinode Flare Catalog, maintained by ISAS/JAXA and Solar-Terrestrial Environment Laboratory, Nagoya University. FJGP would also like to give a special thank for the technical support provided by NOAA/SWPC and NICT SW services and for the valuable remarks of D. Vaz and J. Martins.

\section{References}

[1] Schrjver, C. 2015. Socio-economic hazards and impacts of space weather: the important range between mild and extreme. Space Weather, 13, 9

[2] Wackler, T. 2015. National Science and Technology Council; National Space Weather Strategy Notices, $80(83), 24,29624,297$

[3] William, B. and Chan-Park, C. 2015 The Origin of Space Weather. Space Weather, 3(2), 99-103

[4] Bolduc, L. 2002 GIC observations and studies in the Hydro-Qu bec power system. Journal of Atmospheric and Solar-Terrestrial Physics, 64, 17931802, doi: 10.1016/S1364-6826(02)00128-1. 1

[5] Kelly, M.A. and Comberiate, J.M. and Miller, E.S. and Paxton, L.J. 2014 Progress toward forecasting of space weather effects on UHF SATCOM after Operation Anaconda. Space Weather, 12(10), 601611

[6] National Research Council 2008 Severe Space Weather Events Understanding Societal and Economic Impacts Workshop Report, chap. 5, 5068. National Academies Press

[7] Barata, T. and Martins, P. and Fernandes, J.M. and Duro, N. 2015 A meteorologia espacial. Segurança e Defesa, 31, 6769

[8] Carrington, R. C. 1859 Description of a Singular Appearance seen in the Sun on September 1, 1859. MNRAS, 20,1315, doi: 10.1093/mnras/20.1.13 
[9] Lloyds 2013 Solar storm risk to the North Anerican Electric grid. URL: https://www.lloyds.com/newsand-insight/risk-insight/library/natural-environment/solar-storm

[10] Tobiska, W.K. 2015 Commercial space weather in respose to societal needs. In C. Schrijver, F. Bagenal, and J. Sojka, eds., Heliophysics V. Space weather and society, chap. 3, 4573

[11] Araujo-Pradere, E. A. and Fuller-Rowell, T. J. and Spencer,, P.S.J. and and Minter, C.F. 2007 Differential validation of the USTEC model. Radio Science, 42, RS3016, 2007

[12] Watermann. 2011 Scientific report from the 3rd COST ES0803 workshop Assessment and validation of space weather models, Alcala Spain, 1617 March, URL: www.costes0803.noa.gr/documents/meetings/costes080alcala/ Scientific\%20report\%20Alcala\%20workshop.pdf

[13] Devos, A. and Dumbović, M. and Rodriguez, L. and Bourgoignie, B. and Kraaikamp, E. and et al. 2013 Statistical model for predicting arrival and geoeffectiveness of CMEs based on near realtime remote solar observations, Poster session presented at 10th European Space Weather Week, Antwerp, Belgium

[14] Arge, C. N. and Pizzo, V. J. 2000 Improvement in the prediction of solar wind conditions using near-real time solar magnetic field updates . J. Geophys. Res., 104, 10,465

[15] Odstrcil, D. 2003 Modeling 3-D solar wind structure. Advances in Space Research, 32, 497506

[16] Newell, P. T. and Sotirelis, T. and Wing, S. 2009 Diffuse, monoenergetic, and broadband aurora: The global precipitation budget . J. Geophys. Res., 114, 207

[17] Bergeot, N. and Chevalier, J.-M. and Bruyninx, C. and Pottiaux, E. and Aerts, W. and Baire, Q. and Legrand, J. and Defraigne, P. and Huang, W. 2014 Near real-time ionospheric monitoring over Europe at the Royal Observatory of Belgium using GNSS data. J. Space Weather Space Clim., 4, A31

[18] Benz A. O. and Monstein C. and Mayer H. 2004 CALLISTO - A New Concept for Solar RadioSpectrometers Kluwer Academic Publisher, The Netherland

[19] Watanabe, K. and Masuda, S. and Segawa, T. 2012 Hinode Flare Catalog. Solar Physics, 279, 317322

[20] Mueller, D., Fleck, B., Dimitoglou, G., et al. 2010, 38th COSPAR Scientific Assembly, 38, 8

[21] Wing, S. and Johnson, J. R. and Jen, J. and et al. 2005 J. Geophys. Res., 110(4)

[22] Sauer, H. H. and Wilkinson, D. C. 2008 Global mapping of ionospheric HF/VHF radio wave absorption due to solar energetic protons. Space Weather, 6, S12,002

[23] Florens, M. and Cairns, S. L. and Knock, S. A. and Robinson, P. A. 2007 Data-driven solar wind model and prediction of type II bursts. Geophys. Res. Lett., 34, L04, 104

[24] Shue, J.-H. and Chao, J. K. and Fu, H. C. and Russell , C. T. and Song, P. and Khuran, K. K. and Singer, H. J. 1997 A new functional form to study the solar wind control of the magnetopause size and shape. JGR, 102, 94979511

[25] Baker, D. N. and Kanekal, S. G. and Blake, J. B. 2004 Characterizing the Earths outer Van Allen zone using a radiation belt content index. Space Weather, 2, S02,003

[26] IPS, Radio and Space Services 2009 ASAPS for Windows V5.3 tutorial

[27] Verbeeck, C. and Higgins, P. and Colak, T. and Watson, T. and Delouille, V. and Mampaey, B. and Qahwaji, R. 2013 A Multi-wavelength Analysis of Active Regions and Sunspots by Comparison of Automatic Detection Algorithms. Solar Physics, 283, 6795 
[28] Robbrecht, E. and Berghmans, D. 2004 Automated recognition of coronal mass ejections (CMEs) in near-real-time data. A\&A, 425, 10971106

[29] Bonte, K. and Berghmans, D. and De Groof, A. and Steed, K. and \& Poedts, S. 2013 Sol. Phys., 286, 185

[30] Kraaikamp, E. and Verbeeck, C. 2015 Solar Demon - an approach to detecting flares, dimmings, and EUV waves on SDO/AIA images. Journal of Space Weather and Space Climate, 5(27), A18

[31] Verbeeck, C. and Delouille, V. and Mampaey, B. and De Visscher, R. 2914 The SPoCA-suite: Software for extraction, characterization, and tracking of active regions and coronal holes on EUV images. A\&A, 561, A29

[32] Gissot, S. F. and Hochedez, J.-F. and Dibos, F. and Brajša, R. and Jacques, L. and Berghmans, D. and Zhukov, A. and Clette, F. and Wöhl, H. and Antoine, J.-P. 2003 Extracting the apparent motion from two successive EIT images. In A. Wilson, ed., Solar Variability as an Input to the Earths Environment, vol. 535 of ESA Special Publication, 853856

[33] Hong, S. and Lee, S. and Oh, S. and Kim, J. and Lee, J. and Kim, Y. and Lee, J. and Moon, Y. and Lee, D. 2012 An Automated Solar Synoptic Analysis Software System, AGU Fall Meeting Abstracts

[34] Bailey, G.J. and Sellek, R. 1990 A mathematical model of the Earths plasmasphere and its application in a study of $\mathrm{He}+$ at $\mathrm{L}=3$. Ann. Geophysicae, 8, 171190

[35] Souza, J.R. and Brum, C.G.M. and Abdu, M.A. and Batista, I.S. and Asevedo Junior, W.D. and Bailey, G.J. and Bittencourt, J.A. 2010 Parameterized regional ionospheric model and a comparison of its results with experimental data and IRI representations. Advances in Space Research, 46, 10321038

[36] Lam, H.-L. 2004 On the prediction of relativistic electron fluence based on its relationship with geomagnetic activity over a solar cycle. Journal of Atmospheric and Solar-Terrestrial Physics, 66, 17031714

[37] Saiz, E. and Cid, J. C. and Cerrato, Y. 2008 Forecasting intense geomagnetic activity using interplanetary magnetic field data. Ann. Geophys., 26, 39893998

[38] Aguado, J. and Cid, C. and Saiz, E. and Cerrato, Y. 2010 A Hyperbolic Decay of the Dst Index during the Recovery Phase of Intense Geomagnetic Storms. JGR, 115, A07,220

[39] Tsagouri, I. and Belehaki, A. and Bergeot, N. and Cid, C. and Delouille, V. and Egorova, T. and Jakowski, N. and Kutiev, I. and Mikhailov, and Nuñez, M. and Pietrella, M. Potapov, A. Qahwaji, R. and Tulunay, Y. and Velinov, P. and Viljanen, A. 2013 Progress in space weather modeling in an operational environment. JSWSC, 3, A17

[40] Meehan, J. and Kunches, J. 2012 Workshop Addresses Aviation Community. Space Weather, 10, S08011, 2012.10.1029/2012SW000828

[41] Meier, M. M. and Matthiä, D. A space weather index for the radiation field at aviation altitudes. Journal of Space Weather and Space Climate, 4(27), A13, 2014. 10.1051/swsc/2014010

[42] McIntosh, P.S. 1990 Sol. Phys., 125, 251

[43] Colak, T. and Qahwaji, R. 2009 Space Weather, 7, S06, 001

[44] Dierckxsens, M. and Tziotziou, K. and Dalla, S. and Patsou, I. and Marsh, M. and et al. 2014 COMESEP and the SEP Forecast Tool, 2014. 11th European Space Weather Week, 17-21 of November, Liege, Belgium

[45] Núñez, M. 2011 Predicting solar energetic proton events (E > $10 \mathrm{MeV}$ ). Space Weather, 9, 3 
[46] Baker, D. N. and McPherron, R. L. and Cayton, T. E. and Klebesadel, R. W. 1990 Linear Prediction Filter Analysis of Relativistic Electron Properties at 6.6 Re. J. Geophys. Res., 95, 15,133

[47] Kutiev, I. and Muhtarov, P. and Andonov, B. and Warnant, R. Hybrid model for nowcasting and forecasting the K index. J. Atmos. Sol. Terr.Phys., 71, 589596

[48] Crown, M.D. 2012 Validation of the NOAA Space Weather Prediction Centers solar flare forecasting look-up table and forecaster-issued probabilitie. Space Weather, 10, 6006

[49] Devos, A. and Verbeeck, C. and Robbrecht, E. 2014 Verification of space weather forecasting at the Regional Warning Center in Belgium. J. Space Weather Space Clim., 4, A29

[50] Hathaway, D. H. and Wilson, R. M. and Reichmann, E. J. The shape of the sunspot cycle. Sol. Phys., 151,177190 